\title{
Gastrointestinal risks of incretin-based drugs
}

...use of

incretin-based

drugs was not associated

with an

increased

risk of acute

pancreatitis...

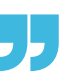

Incretin-based drugs have been linked with an increased risk of acute pancreatitis. However, studies investigating this association have yielded conflicting results and have had major limitations, such as small sample sizes. Now, a large nested case-control study has found no association between the use of incretin-based drugs and acute pancreatitis. Furthermore, a second study found that glucagon-like peptide 1 (GLP1) analogues, but not dipeptidyl peptidase 4 (DPP4) inhibitors, are associated with an increased risk of bile duct and gall bladder diseases.

In the first study, databases from three countries (Canada, the USA and the UK) were used to identify $1,532,513$ patients with type

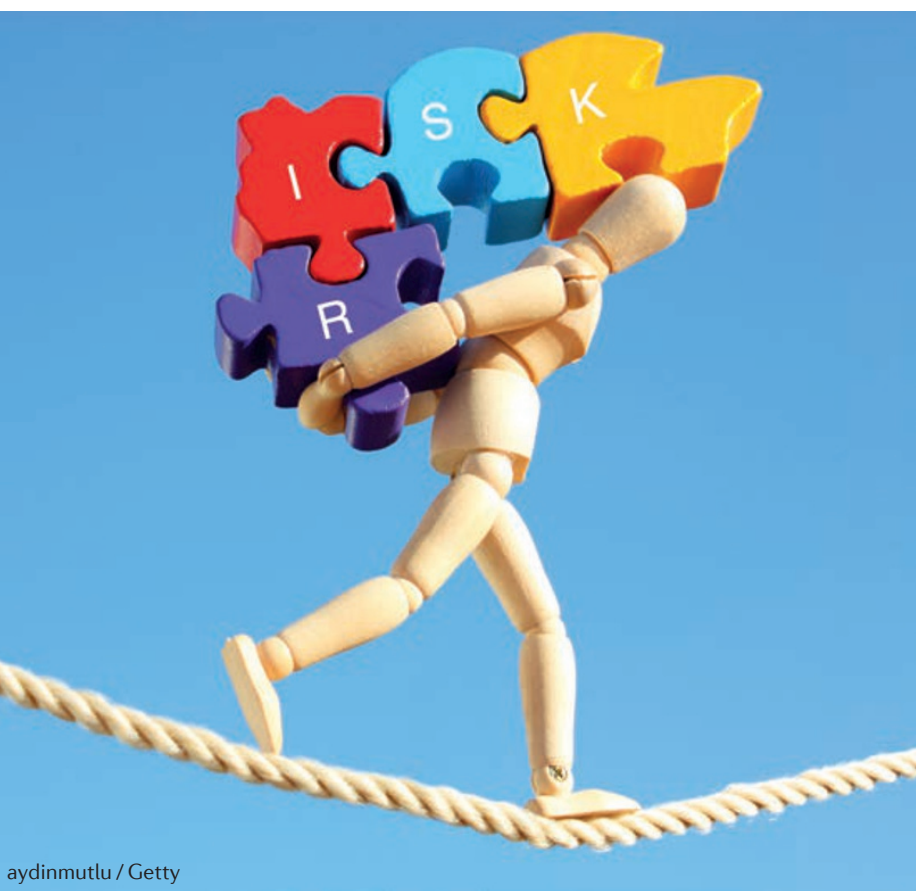

2 diabetes mellitus (T2DM) who started taking antidiabetic drugs (either incretin-based drugs or at least two oral antidiabetic drugs) between 1 January 2007 and 30 June 2013.

Of the patients included in the analysis, 5,165 were hospitalized as a result of acute pancreatitis. Patients who developed acute pancreatitis during the follow-up period (until 30 June 2014) were matched with up to 20 control individuals for features such as age, sex and duration of T2DM treatment. Importantly, the use of incretin-based drugs was not associated with an increased risk of acute pancreatitis, compared with the use of two or more oral antidiabetic agents. The risk did not vary by drug class and no evidence of a duration-response association was found. "We did not find an association between incretin-based drugs and acute pancreatitis, which has been a contentious safety issue for a number of years," says Laurent Azoulay, corresponding author of both papers. "While our study did not find an association, which should provide some reassurance to physicians and patients, it is not possible to rule out a small increased risk."

In the second study, a population-based cohort study, Azoulay and colleagues used a UK database to identify 71,369 patients with T2DM who started taking antidiabetic agents between 1 January 2007 and 31 March 2014. Over the follow-up period (until 31 March 2014), 853 patients were hospitalized for bile duct or gall bladder disease.

The analysis revealed that use of DPP4 inhibitors was not associated with an increased risk of these outcomes, compared with use of two or more antidiabetic oral agents. However, "the use of GLP1 analogues was associated with a $79 \%$ increased risk," explains Azoulay. "We believe that physicians should inform patients about this possible risk and be vigilant when treating patients with these drugs." The authors suggest that the different effects of the two drug classes could be related to their differing pharmacology. DPP4 inhibitors increase the half-life of endogenous GLP1, whereas the GLP1 analogues are direct exogenous agonists to the GLP1 receptor. This direct mode of action leads to a much stronger incretin action than with DPP4 inhibitors.

Azoulay notes that these findings suggest several future avenues for research. "While we did not observe an increased risk of acute pancreatitis with incretin-based drugs in the relative short-term, it will be of interest to re-investigate this association once these drugs have been on the market for a longer period of time," says Azoulay. "As for the bile duct and gall bladder disease study, it remains to be seen whether the risk observed in our study also translates to an increased risk of cancers of the bile duct and gall bladder."

Claire Greenhill

ORIGINAL ARTICLES Azoulay, L. et al.

Association between incretin-based drugs and the risk of acute pancreatitis. JAMA Intern. Med. http:// dx.doi.org/10.1001/jamainternmed.2016.1522 (2016) | Faillie, J.-L. et al. Association of bile duct and gallbladder diseases with the use of incretinbased drugs in patients with type 2 diabetes mellitus. JAMA Intern. Med. http://dx.doi. org/10.1001/jamainternmed.2016.1531 (2016) 